\title{
Assessment of neuropsychological function in brain tumor treatment: a comparison of traditional neuropsychological assessment with app-based cognitive screening
}

\author{
Rafael Romero-Garcia ${ }^{1,2} \mathbb{1} \cdot$ Mallory Owen $^{1} \cdot$ Alexa McDonald $^{3} \cdot$ Emma Woodberry $^{3} \cdot$ Moataz Assem $^{4}$. \\ Pedro Coelho ${ }^{5} \cdot$ Rob C. Morris $^{6} \cdot$ Stephen J. Price ${ }^{6} \cdot$ Tom Santarius $^{6,7} \cdot$ John Suckling $^{1,8,9} \cdot$ Tom Manly $^{9} \cdot$ Yaara Erez $^{4,10}$. \\ Michael G. Hart ${ }^{1}$
}

Received: 9 August 2021 / Accepted: 16 February 2022 / Published online: 1 March 2022

(c) The Author(s) 2022

\begin{abstract}
Background Gliomas are typically considered to cause relatively few neurological impairments. However, cognitive difficulties can arise, for example during treatment, with potential detrimental effects on quality of life. Accurate, reproducible, and accessible cognitive assessment is therefore vital in understanding the effects of both tumor and treatments. Our aim is to compare traditional neuropsychological assessment with an app-based cognitive screening tool in patients with glioma before and after surgical resection. Our hypotheses were that cognitive impairments would be apparent, even in a young and high functioning cohort, and that app-based cognitive screening would complement traditional neuropsychological assessment. Methods Seventeen patients with diffuse gliomas completed a traditional neuropsychological assessment and an app-based touchscreen tablet assessment pre- and post-operatively. The app assessment was also conducted at 3-and 12-month followup. Impairment rates, mean performance, and pre- and post-operative changes were compared using standardized Z-scores. Results Approximately 2-3 h of traditional assessment indicated an average of 2.88 cognitive impairments per patient, while the 30-min screen indicated 1.18. As might be expected, traditional assessment using multiple items across the difficulty range proved more sensitive than brief screening measures in areas such as memory and attention. However, the capacity of the screening app to capture reaction times enhanced its sensitivity, relative to traditional assessment, in the area of non-verbal function. Where there was overlap between the two assessments, for example digit span tasks, the results were broadly equivalent.

Conclusions Cognitive impairments were common in this sample and app-based screening complemented traditional neuropsychological assessment. Implications for clinical assessment and follow-up are discussed.
\end{abstract}

Keywords Glioma $\cdot$ Neuropsychology $\cdot$ Neuropsychiatry $\cdot$ Neurosurgery $\cdot$ Cognitive function

\section{Introduction}

Patients with diffuse glioma can develop impairments in multiple cognitive domains before or after surgery that can have a profound effect on function and quality of life [9,

Rafael Romero-Garcia and Mallory Owen contributed equally to this work.

This article is part of the Topical Collection on Brain Tumors

Rafael Romero-Garcia

rr480@cam.ac.uk

Extended author information available on the last page of the article
25]. The type of impairment can be influenced by tumor morphology, extent of resection and post-surgical treatment, tumor recurrence, age, and concurrent psychological distress $[1,8,13,15,16,19,30,34,35]$. However, in any given case, it is difficult to make precise predictions about cognitive outcome due to, e.g., individual differences and long-ranging effects of diffuse gliomas on networks throughout the brain $[11,12,27,31]$. Assessment of cognitive function is therefore vital in informing management and in monitoring the long-term effects of tumors and interventions.

Despite recognition of this importance, several issues remain with current diffuse glioma neurocognitive testing. In a traditional cognitive assessment, a neuropsychologist works through a range of in-depth assessments with the patient, 
writing down the responses then manually scoring and writing a report. The range of tests reflects the fractionation of the cognitive system, that is distinct functions that can be selectively impaired. For example, in the area of memory, a neuropsychologist may apply different tests of verbal and non-verbal memory, recognition memory vs. recall, autobiographical memory, prospective memory, working memory, and so on. These tests typically present many items, graded in difficulty, such that the tests are sensitive to individual differences in the general population as well as simply detecting "impairment."

This approach has great strengths but also important limitations. In-depth assessment of an individual can take several hours, in addition to scoring and report writing time. A long assessment may be highly sensitive in the general population but be overly influenced by fatigue, pain, or other factors in clinical groups [30]. There is such a wide range of specialized tests available that there may be little overlap between those used in one center and another, making comparison of outcomes complex [22, 26, 28]. Many tests have strong or unknown practice effects, limiting their use for repeated assessments.

Computerized cognitive assessments offer potential advantages including highly standardized administration, the capacity to measure reaction times with millisecond precision, and instant scoring/reporting. In this way, indications of possible impairment in language, memory, orientation, attention, numerical cognition, spatial bias, and ability to imitate gestures can be derived in around $10 \mathrm{~min}$, helping to inform management in settings where patients may be intolerant of longer assessments (e.g., acute stroke). The advent of the touchscreen tablet added portability and suitability for bedside use, greater hygiene, a more intuitive means of interaction, and further reduced costs. OCS-BRIDGE is a recently developed tablet app that is a hybrid between very brief screening and more in-depth assessment (https://ocs-bridge.com/) [6, 18].

To date, there are no reports of the use of app-based cognitive assessment in diffuse glioma. Here 17 patients were given a traditional neuropsychological assessment and OCSBRIDGE assessment before and after surgery. In addition, OCS-BRIDGE was re-administered at 3- and 12-month follow-up. Our interest was whether OCS-BRIDGE offered advantages in terms of ease of administration and efficiency of repeated testing in longitudinal studies, whether its results accorded well with traditional in-depth assessment, and whether aspects of the two approaches may be complementary.

\section{Materials and methods}

\section{Participants}

This study is a single-center prospective cohort design approved by the Cambridge Central Research Ethics
Committee (protocol number 16/EE/0151). All procedures contributing to this work comply with the ethical standards of the relevant national and institutional committees on human experimentation and with the Helsinki Declaration of 1975, as revised in 2008. All patients gave written informed consent. Patients deemed to have typical appearances of a diffuse glioma were identified at adult neuro-oncology multidisciplinary team (MDT) meetings at Addenbrooke's Hospital (Cambridge, UK), and a consultant neurosurgeon directly involved in the study identified potential patients based on the outcome of the MDT discussion. Patients $(n=17)$ were recruited between 2017 and 2019 and followed up until 2020. Inclusion criteria included the following: (i) participant is willing and able to give informed consent for participation in the study, (ii) imaging is evaluated by the MDT and judged to have typical appearances of a diffuse glioma, (iii) stealth MRI is obtained (routine neuronavigation MRI scan performed prior to surgery), (iv) World Health Organization (WHO) performance status 0 or 1, (v) age between 18 and 80 years, (vi) tumor located in or near eloquent areas of the brain thought to be important for speech and executive functions, and (vii) patient undergoing awake surgical resection of a diffuse glioma. This last inclusion criterion was adopted to collect additional intraoperative electrocorticography data that will be reported separately [10]. Participants were excluded if any of the following applied: (i) concomitant anti-cancer therapy, (ii) concomitant treatment with steroids, (iii) history of previous malignancy (except for adequately treated basal and squamous cell carcinoma or carcinoma in situ of the skin) within 5 years, and (iv) previous severe head injury. See Table 1 for demographic and clinical characteristics.

\section{Cognitive assessment}

Cognitive assessment was carried out using two distinct methods at various time points before and after surgery.

\section{Traditional neuropsychological assessment}

The neuropsychological battery comprised 26 independent measures of cognitive function across eight domains: verbal memory, nonverbal memory, verbal skills, nonverbal skills, attention, executive function, and mood disturbance using a variety of previously validated tests $[20,24,33]$. The battery included elements of both the Weschler Adult Intelligence Scale IV, which has previously been used to assess cognitive functioning in glioma patients [37], and the Brain Injury Rehabilitation Trust Memory and Information Processing Battery, which was specifically designed for patients with neurological injuries [5]. Testing took approximately $2-3 \mathrm{~h}$ to complete and was administered by a registered neuropsychologist in a 
Table 1 Demographic information. $S F G$, superior frontal gyrus; $M F G$, middle frontal gyrus; $I F G$, inferior frontal gyrus; ITG, inferior temporal gyrus; $M T G$, middle temporal gyrus; SMA, supplementary motor area. Ages have been rounded to off for anonymity

\begin{tabular}{llllllll}
\hline Patient & Age & Gender & Handedness & Presentation & Hemisphere & Location & Tumor grade/pathology \\
\hline 1 & 40 & Female & Left & Seizures & Left & Frontal & Grade II oligodendroglioma \\
2 & 30 & Male & Right & Seizures & Right & Insula & Grade II astrocytoma \\
3 & 30 & Male & Right & Seizures & Left & Temporal/insula & Grade IV glioblastoma \\
4 & 50 & Female & Right & Incidental & Right & Insula & Grade II oligodendroglioma \\
5 & 60 & Female & Right & Recurrence & Left & Frontal/SFG/frontal pole & Grade II oligodendroglioma \\
6 & 20 & Female & Left & Seizures & Right & Frontal/IFG & Grade I ganglioglioma \\
7 & 30 & Male & Right & Seizures & Right & Frontal/SFG and MFG & Grade III astrocytoma \\
8 & 30 & Male & Right & Seizures & Right & Frontal/MFG & Grade III astrocytoma \\
9 & 50 & Male & Left & Seizures & Left & Temporal/ITG & Grade IV glioblastoma \\
10 & 40 & Female & Right & Seizures & Right & Frontal/MFG & Grade II oligodendroglioma \\
11 & 30 & Male & Right & Seizures & Left & Frontal/SFG/frontal pole & Grade II astrocytoma \\
12 & 30 & Female & Right & Headaches & Left & Temporal/MTG & Grade III astrocytoma \\
13 & 30 & Female & Right & Seizures & Left & Superior temporal gyrus & Grade I ganglioglioma \\
14 & 60 & Female & Right & Seizures & Left & Superior temporal gyrus & Grade II astrocytoma \\
15 & 30 & Male & Right & Seizures & Left & Superior temporal gyrus & Grade III astrocytoma \\
16 & 30 & Male & Right & Seizures & Left & SFG/SMA and pre-central & Grade IV glioblastoma \\
17 & 30 & Male & Right & Seizures & Left & Inferior frontal & Grade III astrocytoma \\
\hline
\end{tabular}

clinical setting. A full list of the tests included on both the OCS-BRIDGE and the neuropsychological assessments can be found in Table $\mathrm{S} 1$.

\section{OCS-BRIDGE assessment}

This novel app-based screening tool, administered via touchscreen tablet, consists of 3 parts. The first part is based on the Oxford Cognitive Screen (OCS) and consists of a brief screening of language, orientation, attention, perception, memory, praxis, and numeracy skills, based on a paperand-pencil measure extensively validated in stroke $[6,18]$. The second part of OCS-BRIDGE provides more sensitive measures, adapted from well-established test paradigms, for patients able to tolerate a slightly longer assessment of 25-40 min. It includes measures of reaction time, working memory, and visual perception. Screening included 6 memory tasks: free verbal memory, overall verbal memory, episodic memory, orientation (spatial memory), forward and backward digit span (verbal short term and working memory) $[32,36]$. Because performance benefits from previous exposure to the tasks (practice effect) tend to be less marked on such measures, they also lend themselves to repeat assessment in longitudinal follow-up. The final part of OCS-BRIDGE consists of the widely used mood measures PHQ-9 and GAD-7 [14, 29]. OCS-BRIDGE was administered pre-operatively, post-operatively prior to discharge, as well as at 3-month and 12-month follow-up, and took between 20 and 35 min to complete.

\section{Cognitive impairment quantification}

An impairment in either assessment technique was defined by convention as performance two standard deviations below the mean of a reference control population on any particular test or test component. OCS-BRIDGE offers a second category of "possibly impaired" for scores that fall approximately between the 5th and the 10th percentile. The total impairments in each domain were defined as the number of individual tests within that domain on which a participant demonstrated an impairment.

Individual domains from the neuropsychological battery and the OCS-BRIDGE assessment were combined into four generalized functional domains for the purposes of direct comparison: attention, memory, verbal skills, and nonverbal skills (Table S2). The neuropsychological battery also included two tests of executive function that provided four independent measures, as well as two assessments of mood. These were included in the analysis as their own domains.

Neuropsychological and OCS-BRIDGE values were $z$-scored by subtracting the mean and dividing by the standard deviation derived from normative healthy participants. Normative data for neuropsychological assessments were obtained from participants aged 16 to 89 . OCS-BRIDGE reference scores were derived from 268 healthy controls (mean age, 51.44, SD age 19.86). These samples were representative of the general distribution in terms of age, gender, and educational level. Neurological/health conditions that were likely to impact on scores (e.g., stroke, epilepsy, medications, uncorrected hearing loss) were an exclusion criterion. 
Further details on the normative data can be found in each individual test manual.

\section{Statistical analysis}

All statistical analyses were carried out in R (R Core Team, 2014) and Matlab R2021b. The $Z$-score of a given domain was calculated as the median $Z$-score of all the items belonging to that domain. We additionally calculated the ratio of cognitive deficits for each patient as the number of tasks showing a deficit (performance below threshold) divided by the total number of tasks. The $t$-value and $P$-value were calculated using an unpaired $t$-test between assessments across patients. The number of tasks that contributed to each domain differed and full details are available in Table S1.

Although the number of participants in the current study is low for formal analysis, for illustration, unsupervised clustering analysis was carried out using the K-means algorithm. Individual tests that did not show any variation in score between participants were omitted from the clustering analysis, excluding 14/39 OCS-BRIDGE, and 1/28 neuropsychological measures. Clustering was performed on values that had been centered to a mean value of 0 with a standard deviation of 1 . K-means clustering was done with a $k$ value of 4 based on the within-groups sum of squares. Analysis was performed using the k-means function in $\mathrm{R}$ version 3.6.1. Clusters were visualized by performing a principal components analysis using the fviz function in the factoextra package and plotting the individual cognitive tests against the first and second principal components.

\section{Results}

\section{Demographics and data completeness}

No significant difference was found in total impairments reported preoperatively between right- and left-handed participants $(t=0.547, P=0.6183)$, male and female $(t=01.175, P=0.258)$, or right- and left-sided tumors $(t=0.845, P=0.411)$. The correlation between age and impairment frequency did not reach statistical significance $(r=-0.22, P=0.39)$.

All participants completed pre-operative traditional neuropsychology and OCS-BRIDGE assessments. Fourteen participants completed traditional neuropsychology between 2 and 5 weeks after surgery, and eight completed postoperative OCS-BRIDGE assessments within $72 \mathrm{~h}$. Eleven participants completed an OCS-BRIDGE assessment at 3-month follow-up and at 12-month follow-up. Sixteen of seventeen participants had at least one OCS-BRIDGE assessment after surgery (see Table S3).

\section{Neuropsychiatric function}

In this sample, anxiety was at a relatively low level with just 3/17 participants pre-operatively scoring within the mild range on the Beck Anxiety Index (BAI) and 2/17 in the moderate range. There was no association between mood status and frequency of cognitive impairment in this sample (BAI status-total cognitive impairments $t=1.2789$, $P=0.22$; BDI status-total cognitive impairments $t=0.87$, $P=0.4)$.

\section{Traditional neuropsychological assessment}

On traditional neuropsychological assessment, pre-surgically, 79\% (14/17) of participants had an impairment in at least one domain, with a mean of $2.88(\mathrm{SD}=2.47)$ impairments per participant. Three participants performed above cutoff in all domains. Impairments were observed in the domains of attention (7), verbal memory (7), verbal skills (6), nonverbal memory (4), and executive function (3). Of the 26 cognitive measures in the battery, 16 detected at least one impaired level of performance among the participants while 10 returned no impaired scores for any participant.

After surgery, all but one participant had an impairment in at least one domain, with a mean of 4.50 impairments per participant $(\mathrm{SD}=3.40)$. Compared with testing before surgery, the total number of cognitive impairments apparently reduced in $43 \%(6 / 14)$ participants, remained unchanged in $7 \%$ (1/14), and increased by $50 \%$ (7/14) (Fig. 1). Of the 7 participants with more impairments, the average increase was 4.14 (SD 3.18). Three domains (verbal memory, nonverbal memory, and verbal skills) demonstrated an increase in impairments after surgery, two domains (nonverbal skills and attention) remained unchanged, and one (executive function) improved. No participants demonstrated an impairment in nonverbal skills at any point.

\section{OCS-BRIDGE testing}

As expected, OCS-bridge assessment required considerably less administration time $($ mean $=30.5 \mathrm{~min}, \mathrm{SD}=7.4)$ than traditional assessment (2-3 h). Before surgery, 59\% $(10 / 17)$ of participants had an impairment in at least one OCS-BRIDGE domain, with a mean of 0.94 impairments $(\mathrm{SD}=1.08)$ per participant. Impairments before surgery occurred in the domains of numerical cognition (4), perception (3), attention (3), language (1), praxis (1), and verbal working memory (1). No participants showed an impairment in memory or prospective memory before surgery. Of the 39 individual cognitive tests included in the OCS-BRIDGE battery, $26(66 \%)$ detected at least 1 impaired performance 
Fig. 1 Change in the total number of deficits for each patient between pre- and postoperative traditional neuropsychology testing

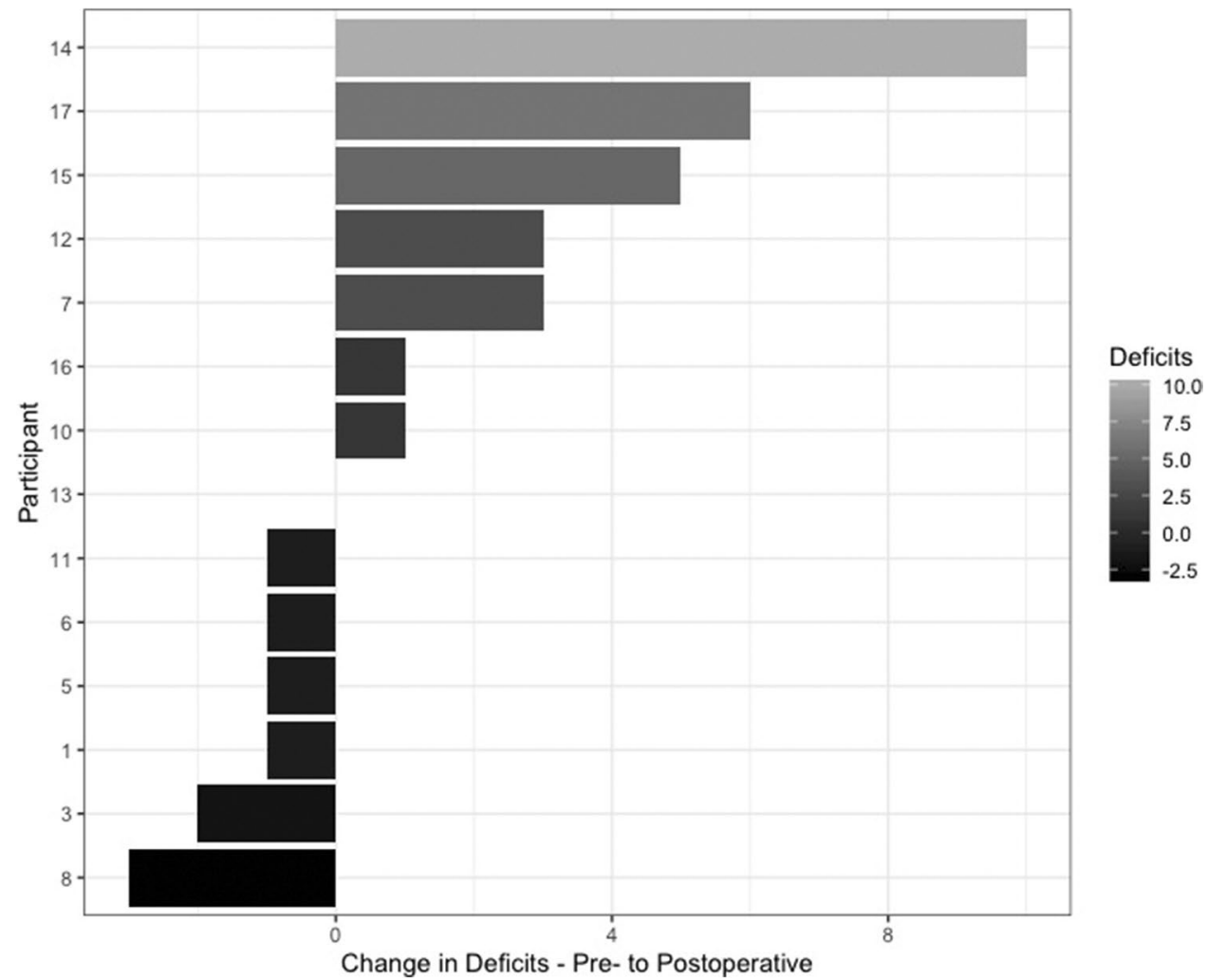

while performance on the remaining 13 was uniformly within the normal range.

\section{OCS-BRIDGE longitudinal changes}

OCS-BRIDGE's relative brevity and ease of administration lent itself to longitudinal assessment as part of follow-up clinics. The variation of postoperative impairments detected is shown in Fig. 2. The greatest number was seen in attention and non-verbal skills. Of the 16 participants who had at least one post-operative assessment, 44\% (7/16) had a reduced number of impairments by their last assessment, 25\% (4/16) had the same, and 31\% (5/16) showed an increase. Four participants who had multiple follow-ups showed a pattern of increased impairments on either post-operative or 3-month testing which resolved by their last follow-up date. Three of these four participants showed a transient increase of at least one impairment in perception, and two showed a transient increase in memory impairments. Ten of the sixteen participants who had at least one follow-up OCS-BRIDGE assessment ended the study with no impairment on formal testing.

\section{Comparison of OCS-BRIDGE with traditional neuropsychological testing}

As discussed, a proportion of the OCS-BRIDGE tests are extremely brief screening measures-the authors, therefore, applied a conservative strategy to avoid a high, and hence uninformative, level of false positives. It would therefore be predicted that OCS-BRIDGE would be less sensitive to impairments than in-depth traditional neuropsychological assessments. Figure 3A shows the total number of preoperative impairments detected in different domains using the two methods (Fig. 3A). The tests in the two batteries vary quite widely but one area of commonality is in the forward and backward digit span tests. As shown in Fig. 3B, the tests returned similar findings with no statistically significant differences on the forward (traditional mean $=6.29, \mathrm{SD}=1.40$ vs. OCS-BRIDGE mean 6.76, SD $=0.90 ; t=-1.367$, $P=0.1905$ ) or backward tests (traditional mean $=4.47$, $\mathrm{SD}=1.28$ vs. OCS-BRIDGE mean $=4.82, \mathrm{SD}=1.55$; $t=-0.972, P=0.3456$ ).

Overall, traditional neuropsychological tests detected 44 preoperative impairments among the 17 participants in the four combined domains of attention, memory, verbal skills, and non-verbal skills. OCS-BRIDGE detected 13 impairments and 28 possible impairments pre-operatively (Fig. 3C). The average ratio of detected deficits was significantly higher for the neuropsychological assessments than for OCS-BRIDGE in the attention (preoperative, $t=3.35$, $P=0.002$; postoperative, $t=2.64, P=0.016$ ) and memory (preoperative, $t=3.24, P=0.003$; postoperative, $t=1.79$, $P=0.088$ ) domains. On the contrary, OCS-BRIDGE revealed a higher ratio of non-verbal skills deficits than 
Fig. 2 Number of cognitive deficits over time based on OCS-BRIDGE assessment preoperatively, postoperatively and at 3- and 12-month follow-ups

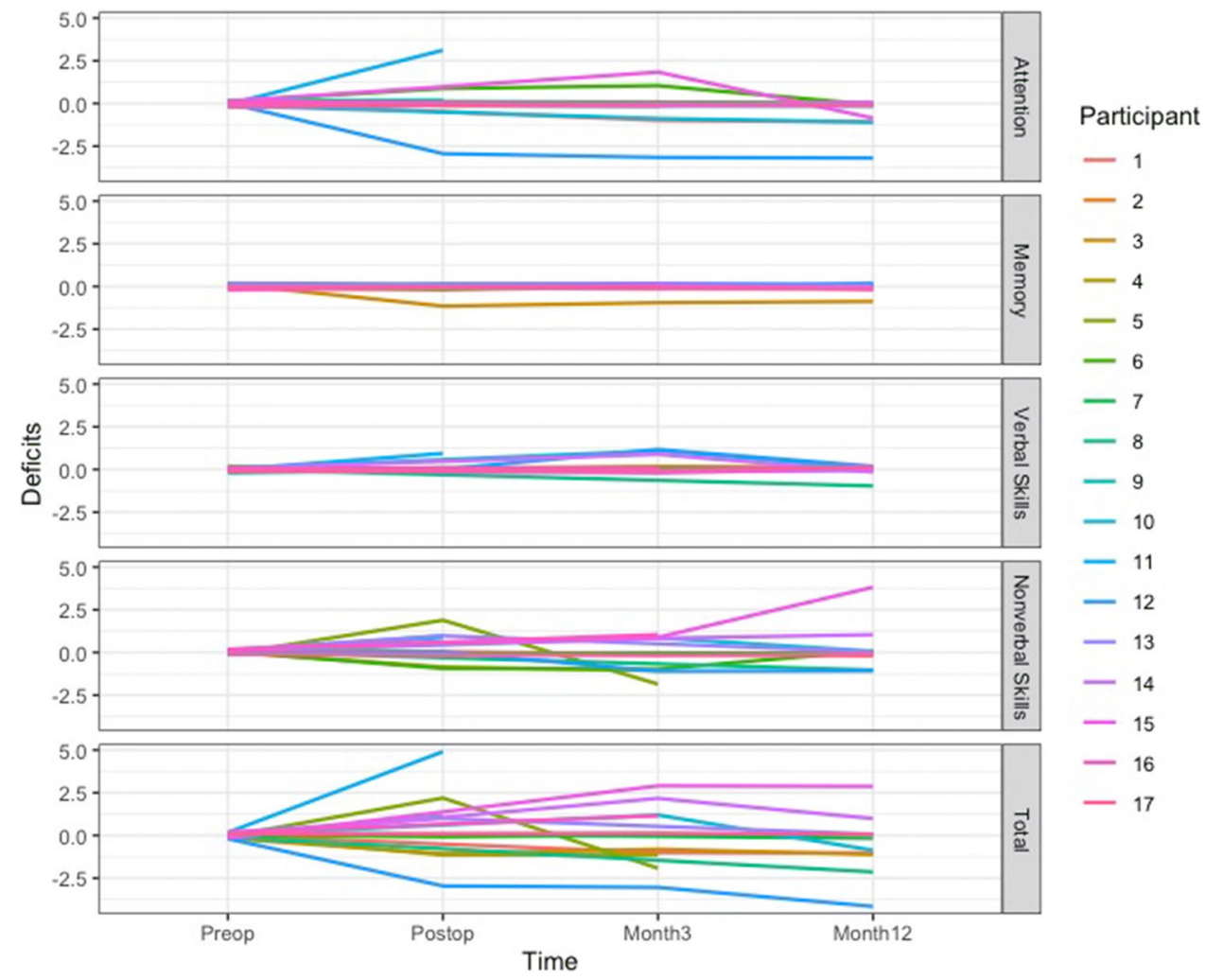

neuropsychological assessments (preoperative, $t=-3.11$, $P=0.004$; postoperative, $t=-3.16, P=0.005$ ) (see Table 2 for the average ratio of deficits per assessment and Table S4 for the total number of deficits).

$Z$-scores were calculated from both the neuropsychological testing and the OCS-BRIDGE data for each individual test (Table 3). The only significant differences before and after surgery at item level (OCS-BRIDGE extinction.totalCorrect and boat.lineHeight; Neuropsychology AIMPB Story Immediate Recall and BMIPB Word list A6) did not survive correction for multiple comparisons. Item $Z$-scores were averaged over each domain, revealing a variety of shape distributions with a predominance of normal (neuropsychology verbal skills) and negatively skewed (memory, non-verbal skills, executive function) forms (Fig. S1). No outliers were observed that may have overtly influenced statistical testing. Total and memory preoperative $Z$-score for neuropsychological testing were weakly correlated with OCS-BRIDGE $Z$-score across patients but significance did not survive multiple correction (Total, $R=0.49$, $P_{\text {uncorrected }}=0.045$; Attention, $R=0.27, P_{\text {uncorrected }}=0.29$; Memory, $\mathrm{R}=0.50, P_{\text {uncorrected }}=0.041$; Non-verbal skills, $R=0.06, P_{\text {uncorrected }}=0.82$; Fig. 4). At the group level, only attention preoperative scores were significantly different between neuropsychology and OCS-BRIDGE $\left(P_{\text {corrected }}=0.04\right.$. Table 4$)$. To explore this further, unsupervised clustering was carried out to assess whether individual tests clustered into groups that were different from the original domains (Fig. 5). Clusters were named for their dominant test type. Cluster 1 included 10 tests, predominantly memory tests from the traditional battery, and included all but one test from AMIPB and BMIPB. Cluster 2 included 10 tests, 8 from the traditional battery and 2 from OCS-BRIDGE, and was mostly composed of Attention and Verbal Skills tests. Cluster 3 included only 4 tests, 3 of which were from the SALT attention task within the OCS-BRIDGE battery. Finally, cluster 4 was the largest cluster, with 29 of the total tests, and included all but one of the tests of non-verbal skills, as well as 10 out of 14 of the OCS-BRIDGE attention tests. Tasks which included several individual tests, such as the OCS-BRIDGE Hearts and SALT tasks, clustered together, in cluster 4 and cluster 3 respectively. The Beck Depression Inventory fell into cluster 1, while the Beck Anxiety Inventory fell into cluster 4.

\section{Discussion}

Diffuse glioma can cause cognitive impairments and identifying these is important in informing patients and families, in improving management, and in evaluating outcomes. Traditional neuropsychological testing is resource-intensive, making it difficult to perform at multiple time points during the patient journey [30]. Tablet-based assessments tools such 
Fig. 3 A Cognitive deficits by domain from preoperative neuropsychological testing and OCS-BRIDGE assessments. B Comparison of digit span analysis between neuropsychological testing and OCS-BRIDGE.

C Total preoperative deficits shown by neuropsychological testing and OCS-BRIDGE

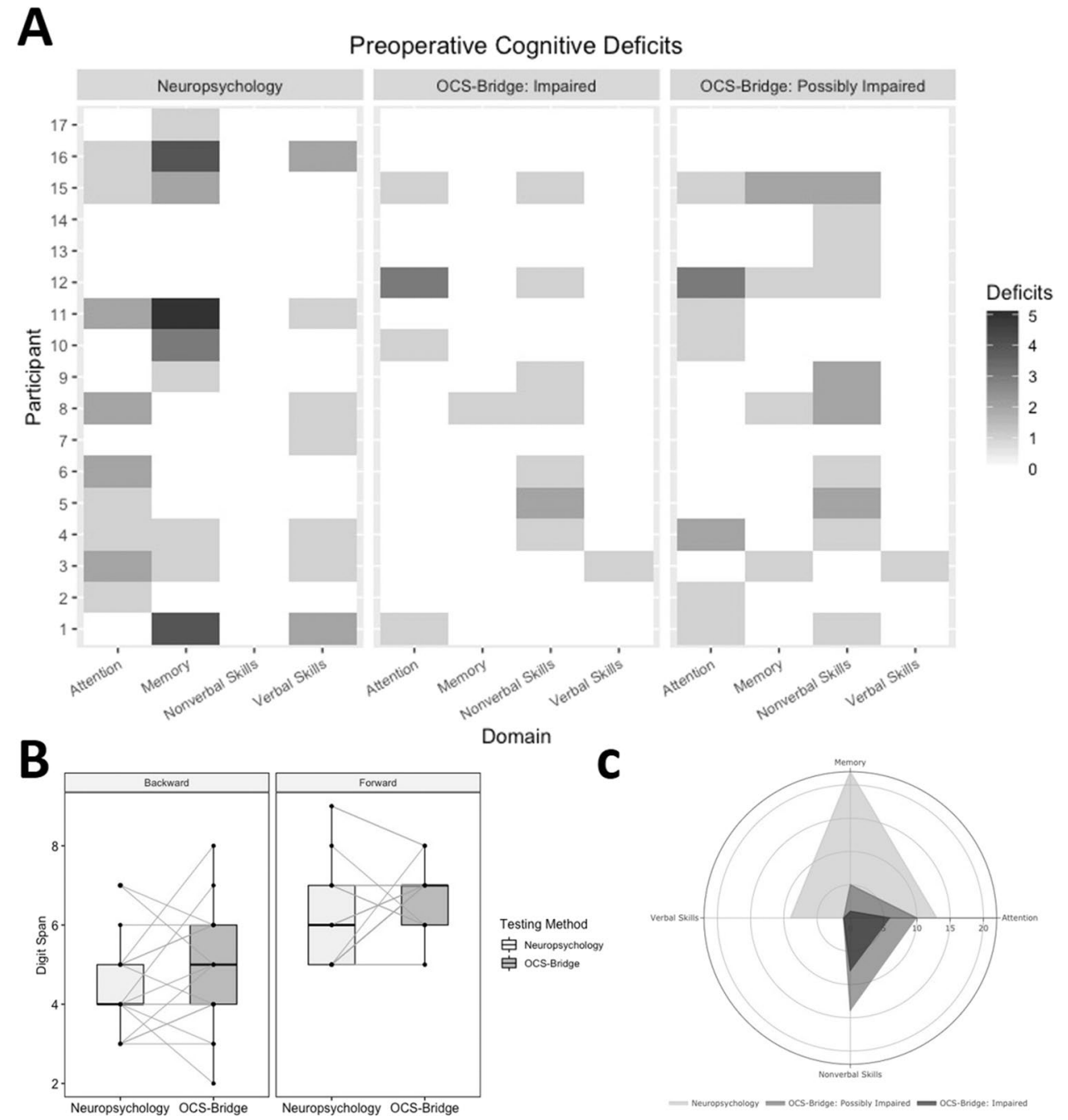

\begin{tabular}{llllll}
\hline & & $\begin{array}{l}\text { Neuropsychology } \\
\text { average ratio (SD) }\end{array}$ & $\begin{array}{l}\text { OCS-BRIDGE } \\
\text { average ratio (SD) }\end{array}$ & $T$ value & $P$ value \\
\hline Attention & Preoperative & $0.255(0.28)$ & $0.025(0.56)$ & 3.35 & $\mathbf{0 . 0 0 2}$ \\
& Postoperative & $0.262(0.23)$ & $0.036(0.76)$ & 2.64 & $\mathbf{0 . 0 1 6}$ \\
\multirow{2}{*}{ Memory } & Preoperative & $0.078(0.10)$ & $0(0)$ & 3.24 & $\mathbf{0 . 0 0 3}$ \\
& Postoperative & $0.131(0.14)$ & $0.031(0.09)$ & 1.79 & 0.088 \\
& Preoperative & $0(0)$ & $0.034(0.04)$ & -3.11 & $\mathbf{0 . 0 0 4}$ \\
& Postoperative & $0(0)$ & $0.080(0.10)$ & -3.16 & $\mathbf{0 . 0 0 5}$ \\
\hline
\end{tabular}

A reoperative Cognitive Deficits

B

$$
\text { Domain }
$$

Table 2 Average ratio and standard deviation of cognitive deficits across patients for each domain and assessment. A positive $T$ value indicates a higher ratio of deficits detected by traditional neuropsychology compared with OCS-BRIDGE as OCS-BRIDGE provide rapid, easy-to-use alternatives to traditional pen-and-paper testing, yet little work has been done to demonstrate the validity of these tests in this population, or to assess their relative strengths and weaknesses when compared with traditional batteries. This paper presents early data from a cohort of 17 participants with diffuse glioma who underwent traditional and OCS-BRIDGE assessment, both before and after surgery, and at several follow-ups. The results show that both methods detected cognitive impairments in participants who did not primarily present with self-reported cognitive problems.

Results revealed that, when compared with a 2-3-h traditional assessment, a 30-min OCS-BRIDGE assessment significantly detected fewer impairments in the areas of attention and memory. However, OCS-BRIDGE significantly detected more impairments in non-verbal skills. Memory and attention screening using only a few items cannot make the kind of fine distinctions possible with a longer test, and 
Table 3 Raw, standard deviation (SD), and Z-scored performance of each item included in traditional neuropsychology and OCS-BRIDGE. $T$-values and $P$-values result from a paired $t$-tests comparing scores before (preop) and after (postop) surgery

\begin{tabular}{|c|c|c|c|c|c|c|c|}
\hline & Item name & $\mathrm{Raw}_{\text {preop }}(\mathrm{SD})$ & $Z_{\text {preop }}$ & $\operatorname{Raw}_{\text {postop }}(\mathrm{SD})$ & $Z_{\text {postop }}$ & $t$-value & $P$-value \\
\hline \multicolumn{8}{|l|}{ OCS-BRIDGE } \\
\hline \multirow[t]{10}{*}{ Attention } & hearts.overallScore & $29.31(-1.08)$ & 0.34 & $28.63(-1.41)$ & -0.06 & 1.33 & 0.20 \\
\hline & hearts.spaceAsymmetry & $-0.002(-0.03)$ & 0.03 & $0(-0.07)$ & 0.10 & -0.19 & 0.85 \\
\hline & hearts.objectAsymmetry & $0.12(-0.6)$ & 0.11 & $0.38(-0.74)$ & 0.55 & -0.93 & 0.36 \\
\hline & hearts.perseverativeResponsesTotal & $0(0)$ & 0.06 & $0(0)$ & 0.06 & 0.00 & 1.00 \\
\hline & hearts.organisationIndex & $-2.64(-0.76)$ & 0.16 & $-2.13(-0.63)$ & 0.70 & -1.64 & 0.11 \\
\hline & salt.overallFlashScore & $3.53(-0.72)$ & -0.02 & $3.43(-0.79)$ & -0.16 & 0.30 & 0.76 \\
\hline & salt.numberAllTargetsDetected & $35.71(-0.69)$ & 0.18 & $35.29(-1.5)$ & -0.28 & 0.96 & 0.35 \\
\hline & salt.RTCoefficient & $-0.15(-0.06)$ & 0.81 & $-0.15(-0.03)$ & 0.90 & -0.26 & 0.80 \\
\hline & salt.targetBias & $0(-0.02)$ & -0.06 & $0(-0.01)$ & 0.22 & -0.66 & 0.52 \\
\hline & salt.RTBias & $0.01(-0.04)$ & 0.17 & $0.04(-0.04)$ & 0.68 & -1.21 & 0.24 \\
\hline \multirow[t]{7}{*}{ Perception } & extinction.totalCorrect & $17.94(-0.24)$ & 0.12 & $17.43(-0.79)$ & -1.30 & 2.48 & 0.02 \\
\hline & extinction.fieldProblem & $0(0)$ & -0.02 & $-0.14(-0.38)$ & -0.61 & 1.61 & 0.12 \\
\hline & extinction.leftExtinction & $0(0)$ & -0.07 & $0(0)$ & -0.07 & 0.00 & 1.00 \\
\hline & extinction.rightExtinction & $0(0)$ & -0.08 & $0.14(-0.9)$ & 0.34 & -0.68 & 0.51 \\
\hline & boat.lineHeight & $-0.99(-0.23)$ & -0.78 & $-1.26(-0.23)$ & -2.21 & 2.70 & 0.01 \\
\hline & boat.meanDeviation & $-0.56(-2.29)$ & -0.11 & $-0.4(-1.4)$ & -0.05 & -0.18 & 0.86 \\
\hline & pstl.overallScore & $6(0)$ & 0.13 & $6(0)$ & 0.13 & 0.00 & 1.00 \\
\hline \multirow[t]{8}{*}{ Memory } & memory.verbalFreeRecall & $3.06(-0.97)$ & -0.18 & $3(-1.51)$ & -0.25 & 0.12 & 0.91 \\
\hline & memory.verbalOverallRecall & $3.88(-0.33)$ & 0.02 & $3.75(-0.46)$ & -0.34 & 0.82 & 0.42 \\
\hline & memory.episodicMemoryScore & $4(0)$ & 0.18 & $4(0)$ & 0.18 & 0.00 & 1.00 \\
\hline & orientation.overallScore & $4(0)$ & 0.17 & $3.88(-0.35)$ & -0.61 & 1.49 & 0.15 \\
\hline & fins.forwardDigitSpan & $6.76(-0.9)$ & -0.20 & $6.14(-1.07)$ & -0.66 & 1.46 & 0.16 \\
\hline & fins.backwardDigitSpan & $4.82(-1.55)$ & -0.39 & $4.43(-1.4)$ & -0.65 & 0.58 & 0.57 \\
\hline & pstp.prospectiveScore & $4(0)$ & 0.71 & $4(0)$ & 0.71 & 0.00 & 1.00 \\
\hline & pstp.retrospectiveScore & $4(0)$ & 0.68 & $4(0)$ & 0.68 & 0.00 & 1.00 \\
\hline \multirow[t]{3}{*}{ Language } & Naming.overallScore & $4(0)$ & 0.32 & $4(0)$ & 0.32 & 0.00 & 1.00 \\
\hline & semantics.overallScore & $3(0)$ & 0.06 & $3(0)$ & 0.06 & 0.00 & 1.00 \\
\hline & reading.overallScore & $15(0)$ & 0.34 & $15(0)$ & 0.34 & 0.00 & 1.00 \\
\hline \multirow[t]{3}{*}{ Praxis } & imitation.handScore & $5.88(-0.49)$ & 0.00 & $5.88(-0.35)$ & -0.02 & 0.04 & 0.97 \\
\hline & imitation.fingerScore & $6(0)$ & 0.20 & $6(0)$ & 0.20 & 0.00 & 1.00 \\
\hline & imitation.overallScore & $11.88(-0.49)$ & 0.12 & $11.88(-0.35)$ & 0.11 & 0.04 & 0.97 \\
\hline \multirow[t]{2}{*}{ Number } & numerical.overallArithmeticScore & $3.47(-0.94)$ & -0.87 & $3.63(-0.52)$ & -0.50 & -0.43 & 0.67 \\
\hline & numerical.overallWritingScore & $2.88(-0.49)$ & -1.00 & $2.88(-0.35)$ & -1.07 & 0.04 & 0.97 \\
\hline \multicolumn{8}{|c|}{ Neuropsychological Assessments } \\
\hline \multirow[t]{3}{*}{ Attention } & WAIS-IV.Digit.Span.Forward & $9.88(-2.5)$ & -0.04 & $9.5(-2.24)$ & -0.17 & 0.44 & 0.66 \\
\hline & WAIS-IV.Digit.Span.Backward & $8.12(-2.2)$ & -0.63 & $7.21(-1.85)$ & -0.93 & 1.22 & 0.23 \\
\hline & WAIS-IV.Digit.Symbol & $66.29(-13.85)$ & -0.18 & $66.07(-15.43)$ & -0.19 & 0.04 & 0.97 \\
\hline \multirow[t]{4}{*}{ Non-verbal skills } & BMIPB.Complex.Figure.copy & $79.12(-1.17)$ & 0.30 & $79.38(-0.87)$ & 0.40 & -0.69 & 0.50 \\
\hline & VOSP.Object.Decision & $18.29(-1.16)$ & -0.19 & $19(-1.22)$ & 0.25 & -1.61 & 0.12 \\
\hline & VOSP.Number.Location & $9.41(-0.87)$ & 0.01 & $9.38(-0.65)$ & -0.01 & 0.09 & 0.93 \\
\hline & VOSP.Cube.Analysis & $9.76(-0.56)$ & 0.39 & $9.77(-0.44)$ & 0.39 & -0.02 & 0.98 \\
\hline
\end{tabular}


Table 3 (continued)

\begin{tabular}{|c|c|c|c|c|c|c|c|}
\hline & Item name & $\mathrm{Raw}_{\text {preop }}(\mathrm{SD})$ & $Z_{\text {preop }}$ & $\operatorname{Raw}_{\text {postop }}(\mathrm{SD})$ & $Z_{\text {postop }}$ & $t$-value & $P$-value \\
\hline \multirow[t]{12}{*}{ Memory } & AMIPB.Story-Immediate.Recall & $34.71(-8.99)$ & 0.17 & $27(-9.04)$ & -0.59 & 2.37 & 0.02 \\
\hline & AMIPB.Story-Delayed.Recall & $32.82(-11.14)$ & 0.18 & $27.54(-11.4)$ & -0.32 & 1.28 & 0.21 \\
\hline & BMIPB.Word.List.A1-A15 & $54.59(-9.1)$ & -0.22 & $50.14(-9.36)$ & -0.84 & 1.34 & 0.19 \\
\hline & BMIPB.Word.List.A6 & $12.82(-2.53)$ & 0.29 & $10.5(-2.95)$ & -0.64 & 2.36 & 0.03 \\
\hline & $\begin{array}{l}\text { BMIPB.Word.List } \\
\text { Word.Recognition }\end{array}$ & $28.71(-0.99)$ & 0.08 & $27.64(-2.47)$ & -0.74 & 1.63 & 0.11 \\
\hline & $\begin{array}{l}\text { BMIPB.Word.List } \\
\text { List.Recognition }\end{array}$ & $28.94(-1.56)$ & -0.04 & $27.5(-3.61)$ & -1.00 & 1.49 & 0.15 \\
\hline & $\begin{array}{l}\text { BMIPB.Complex.Figure } \\
\text { Immediate.Recall }\end{array}$ & $65.47(-7.8)$ & 0.02 & $61.36(-13)$ & -0.39 & 1.09 & 0.28 \\
\hline & $\begin{array}{l}\text { BMIPB.Complex.Figure } \\
\text { Delayed.Recall }\end{array}$ & $63.24(-8.21)$ & 0.11 & $59.57(-13.36)$ & -0.23 & 0.94 & 0.36 \\
\hline & BMIPB.Design.Learning-A1-A15 & $37.35(-6.19)$ & -0.01 & $35.14(-7.22)$ & -0.38 & 0.92 & 0.37 \\
\hline & BMIPB.Design.Learning-A6 & $8.29(-1.16)$ & 0.07 & $8.14(-1.61)$ & -0.04 & 0.30 & 0.76 \\
\hline & $\begin{array}{l}\text { BMIPB.Design.Learning } \\
\text { Recognition }\end{array}$ & $39.41(-1.18)$ & 0.32 & $38.43(-2.59)$ & -0.12 & 1.40 & 0.17 \\
\hline & $\begin{array}{l}\text { BMIPB.Design.Learning } \\
\text { Identification }\end{array}$ & $9.59(-1)$ & 0.13 & $9(-1.62)$ & -0.27 & 1.24 & 0.22 \\
\hline \multirow[t]{4}{*}{ Language } & Letter.Fluency & $37.82(-14.55)$ & -0.25 & $30.71(-15.04)$ & -0.91 & 1.33 & 0.19 \\
\hline & Semantic.Fluency & $20.71(-5.92)$ & 0.22 & $16.5(-5.68)$ & -0.79 & 2.00 & 0.05 \\
\hline & Graded.Naming.Test & $19.65(-3.87)$ & -0.66 & $19.57(-5)$ & -0.68 & 0.05 & 0.96 \\
\hline & Syntactic.Speech.Comprehension & $24.88(-0.93)$ & -0.08 & $24.57(-1.99)$ & -0.29 & 0.57 & 0.57 \\
\hline \multirow[t]{4}{*}{ Executive functioning } & Hayling.Initiation.(time) & $-3.88(-3.76)$ & -0.76 & $-2.5(-1.61)$ & -0.13 & -1.28 & 0.21 \\
\hline & Hayling.Inhibition.(time) & $-21.24(-16.06)$ & -0.36 & $-23(-21.01)$ & -0.49 & 0.27 & 0.79 \\
\hline & Hayling.Inhibition.(score) & $10.94(-3.23)$ & -0.04 & $10.86(-3.72)$ & -0.07 & 0.07 & 0.95 \\
\hline & Brixton & $-10.94(-4.49)$ & 0.89 & $-10.92(-7.1)$ & 0.89 & -0.01 & 0.99 \\
\hline
\end{tabular}

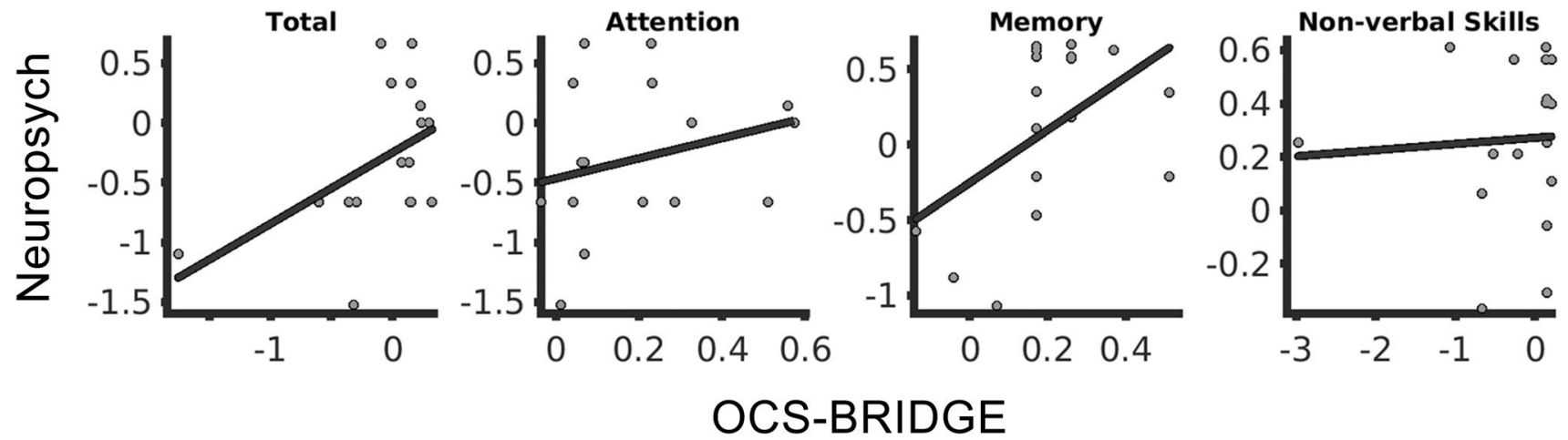

Fig. 4 Association between Z-scores derived from OCS-BRIDGE and neuropsychological testing. Plots for overlapping domains (Attention, Memory, and Non-verbal skills) and for the total average across domains (Total)

as a consequence can have a more conservative threshold for impairment. In contrast, OCS-BRIDGE measures with reaction time assessments can make very fine individual difference distinctions. The results suggest that a combined approach, using traditional assessment in those areas where brief screening, may be less sensitive, and OCS-BRIDGE style measures for reaction time and perceptual tasks may be most effective. 
Table 4 Differences in cognitive performance between traditional neuropsychology and OCS-BRIDGE. $Z_{\text {neuropsych }}$ and $Z_{\text {OCS-bridge }}$ indicate the average $Z$-score cognitive performance and standard deviation (SD) of each domain assessed by neuropsychological testing and OCS-bridge screening respectively

Fig. 5 K-means clustering of individual cognitive tests and validation against deficit numbers. A Principal components analysis plotted versus the k-means clustering results. B Cognitive deficits for each participant by cluster, both before and after surgery

\begin{tabular}{llllll}
\hline & & $Z_{\text {neuropsych }}(\mathrm{SD})$ & $Z_{\text {OCS-bridge }}(\mathrm{SD})$ & $T$-value & Corrected $P$-value \\
\hline Attention & Preoperative & $-0.30(-0.61)$ & $0.19(0.20)$ & -3.47 & 0.0376 \\
& Postoperative & $-0.39(-0.53)$ & $0.18(0.20)$ & -2.49 & 0.1661 \\
\multirow{3}{*}{ Memory } & Preop-postop & $0.09(-0.58)$ & $0.05(0.23)$ & 0.63 & 0.6938 \\
& Preoperative & $0.11(-0.58)$ & $0.21(0.16)$ & -0.77 & 0.6746 \\
& Postoperative & $0.21(-0.47)$ & $0.24(0.14)$ & -0.17 & 0.8746 \\
\multirow{3}{*}{ Non-verbal skills } & Preoperative & $0.26(-0.30)$ & $0.28(0.80)$ & 2.69 & 0.0974 \\
& Postoperative & $0.20(-0.35)$ & $0.11(0.44)$ & 2.75 & 0.1619 \\
\multirow{3}{*}{ Total } & Preop-postop & $-0.03(-0.34)$ & $0.18(0.69)$ & 1.53 & 0.4461 \\
& Preoperative & $-0.11(-0.80)$ & $0.02(0.42)$ & -0.44 & 0.7283 \\
& Postoperative & $-0.18(-0.76)$ & $0.03(0.31)$ & 0.88 & 0.6746 \\
& Preop-postop & $0.12(-0.80)$ & $0.07(0.38)$ & -1.17 & 0.5915 \\
\hline
\end{tabular}

A

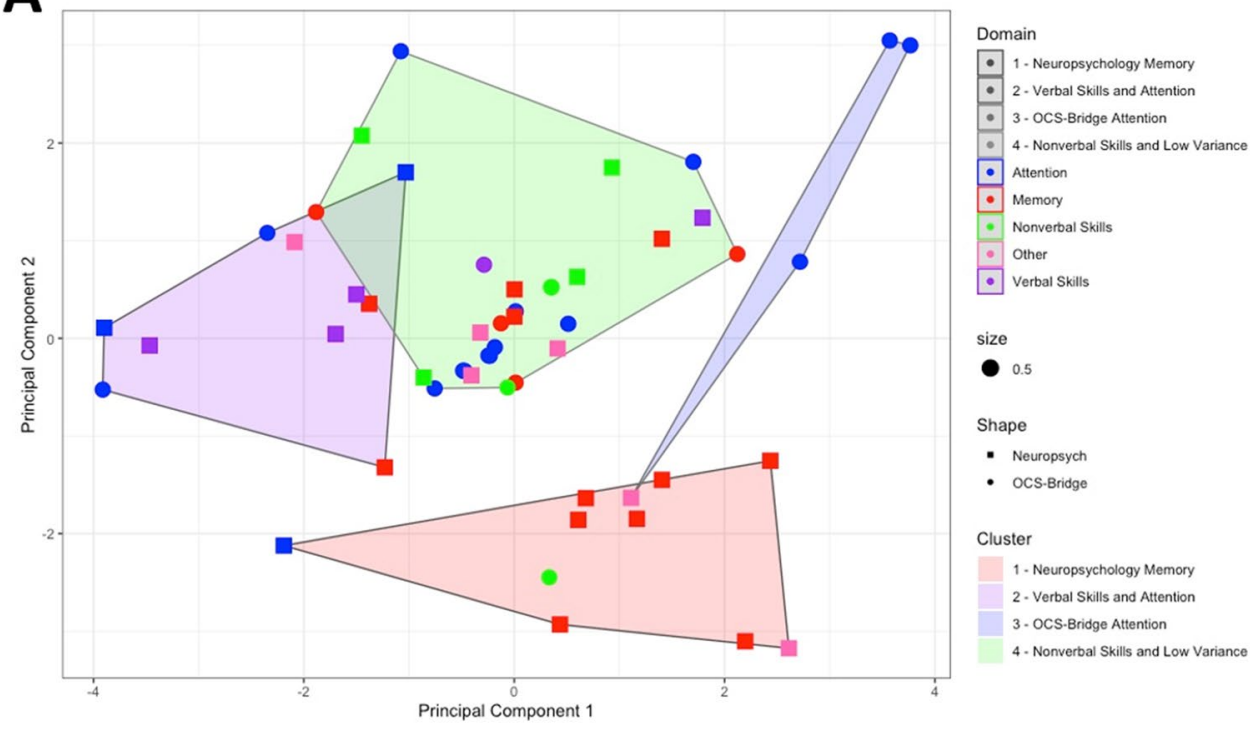

B

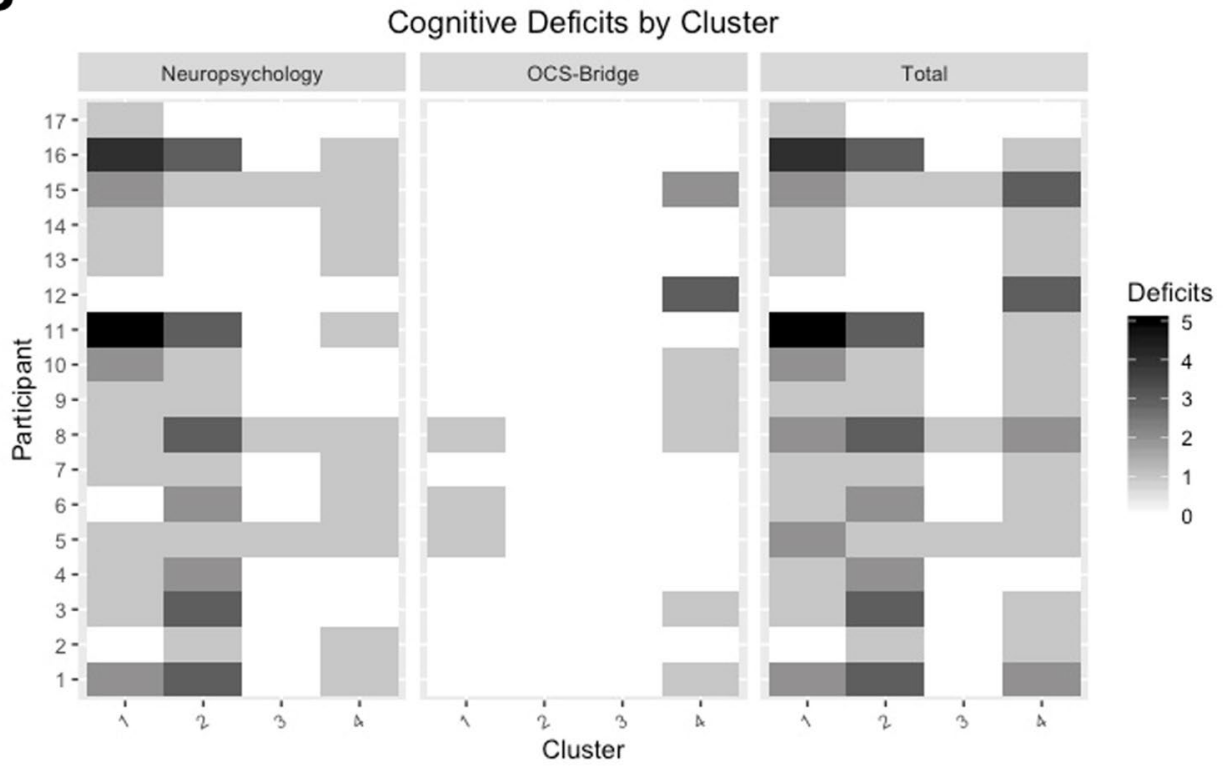


Comparisons based on $Z$-scores showed no significant differences between preoperative and postoperative scores at the individual item level, revealing that single items have insufficient statistical power for detecting average decreases in performance even for individuals undergoing major surgery. However, the lack of significance may be driven by our limited sample size or by the challenges of correcting for multiple comparisons when a large number of tests are considered. Additionally, by grouping items into larger domains, we were able to compare assessment methods. The positive, but nonsignificant after correction, correlation between OCS-BRIDGE and neuropsychological assessment suggest an effect that may be confirmed in larger samples. Nevertheless, the low explained variance is undoubtedly reflecting some degree of disagreement that in turn suggests a lower power for group-based $Z$-scores that compare assessments based on means and standard deviations obtained from patients. This could be particularly challenging for a brain tumor population with highly heterogeneous cognitive profiles. As this $Z$-scored analysis retains the variability of scores even for patients' performances that are within normative ranges (i.e., an unthresholded approach), it may have masked the differences in low-performance domains shown by the analysis based on the number of detected deficits, as discussed above. Thought needs to be given to the practicalities, duration, and tolerability of the assessment as well as the sensitivity of the measures in finding the most appropriate balance for particular clinical groups or individuals.

\section{Advantages of OCS-BRIDGE}

There are several advantages of using a tablet-based system in conjunction with traditional neuropsychological testing in glioma patients. OCS-BRIDGE is easy to transport and can be administered anywhere that is relatively quiet and free from distraction. It is easy to administer in a standardized way without the necessity of a highly trained neuropsychologist at the point of administration. It automatically captures patient responses and scores and reports on performance saving time and reducing errors. Ease of use also presents the possibility that these assessments could be modified to be performed by the patient independently, furthering their potential for tracking subtle changes in cognitive functioning over time during recovery. As is observed here, an app-based system may pick up subtle differences in certain areas of performance missed by traditional testing, such as precise reaction times and accurate visual acuity scores.

\section{Complementary role of traditional and app-based assessment}

Cluster analyses have been extensively exploited in neuropsychology to better understand the relationship between test scores and patient classification [3, 21]. Here, we use this methodology to show that items are grouped not only according to a domain criterion but also following an assessment criterion (traditional vs. app-based). The latter is not surprising given that traditional neuropsychological testing exhibited higher sensitivity to impairments in memory. There are no inherent barriers to recreating indepth memory assessments in app form, as is illustrated by the digit span measures that, in both cases here, began at a very easy level and continued until a participant consistently failed. The clustering also identified a subset of items with very limited sensitivity for detecting impairments (cluster 3 ), suggesting that this could be potentially exploited for discriminating relevant assessments. The OCS memory measures were primarily developed for extremely fast screening in an acute stroke setting and may indeed be less suitable for the diffuse glioma population. In contrast, a tablet's ability to capture reaction time with millisecond precision gives OCS-BRIDGE some sensitivity advantages over traditional neuropsychological assessment. The current results would certainly suggest that combining the strengths of app and traditional approaches would be warranted in the diffuse glioma population (or that more suitable app measures of memory are used). An issue here is whether to tailor a diffuse glioma battery to the time when they may be most fatigued/intolerant of a long testing session (post-surgery) or to shorten/lengthen testing as appropriate for each stage.

Quite a few measures in the two batteries used here had ceiling effects; that is, no participant scored less than perfectly. The reason for their inclusion was that there are important neuropsychological conditions to which such tests are sensitive that may have arisen in a larger sample. In selecting tests for a particular clinical population, the risks of failing to detect an impairment must be weighed against the costs and time of giving that assessment. In that respect, studies such as this are useful for informing such decisions.

Another factor supporting a complementary is the importance of the neuropsychologist, rather than the particular tests used. As discussed, uninterpreted test results can encourage a binary view that a function is either impaired or unimpaired. In contrast, it is possible that an individual who has fallen from the 95th to 15th percentile on a particular test has likely suffered a significant impairment regardless of a test finding performance to be in the "normal range." Neuropsychologists use interviews about educational and occupational history, the experiences of patients and family members, and the patterns across tests to think about what scores are likely to mean and the implications for management, rehabilitation, and so on. There is currently no app for that. It is important that staff using app-based assessments have appropriate training in what the results do, and do not, mean. 
The results presented above indicate potentially complementary roles of different modalities of cognitive testing in the assessment and postoperative follow-up of glioma patients. Traditional testing administered in a clinical setting by a qualified neuropsychologist demonstrated high sensitivity to cognitive impairments, particularly in the domains of memory and verbal skills, when compared with the OCS-BRIDGE tablet-based system. The tablet-based system showed higher sensitivity to impairments in nonverbal skills including impairments in perception, and took less time, expertise, and resources to administer and analyze. These differences indicate that there may be a role for a combined assessment schedule using both modalities for glioma patients in the future to gain a more comprehensive understanding of their functional abilities pre- and postoperatively and as they recover in the longer term.

Combining cognitive assessments in an optimal way represents a major challenge. Existing literature has demonstrated that multiple tests used together can facilitate the diagnosis of neurodevelopmental conditions [7, 23]. However, the criteria for distinguishing clinical differences through battery test combination remains an open question. A liberal threshold considering deficits as those domains showing just at least one impairment in a test would have higher sensitivity and worse specificity than more conservative thresholds. Notwithstanding, Lovejoy et al. [17] showed that a liberal threshold of deficits in at least one out of six tests as signifying impairment provides the best overall classification rates of ADHD (i.e., the improvement in specificity does not outweigh the decrease in sensitivity). This is also of major relevance in our cohort of brain tumor patients where sensitivity is more critical than specificity (as patients with nonidentified deficits-false negativeswill not receive appropriate training or treatment while the contrary does not risk significant harm to the patient). Although systematic comparisons will be necessary to optimally combine traditional neuropsychological and app-based assessments, the combination will need to be tailored to each neuropsychiatric condition and should balance sensitivity and specificity according to the impact that false positives and false negatives have on patients.

\section{Study limitations and constraints on generality}

Beyond the reduced sample size, attrition represents an additional limitation of this study. In particular, the immediate post-operative assessment was often limited by fatigue, while follow-up data was on occasion precluded by the participants' geographical distance from centralized assessment. In a related manner, there were logistical and technical limitations to the assessments, which is not entirely unexpected during the beta-testing phase and with such an ambitious study protocol.
All patients had the pre-operative imaging appearances of a diffuse glioma (non-enhancing and without edema or mass effect); however, subsequent pathological examination revealed a range of histological diagnoses (Table 1). There were other sources of heterogeneity such as tumor location, tumor size, and treatment-some participants received only surgical intervention while others received follow-up chemotherapy or radiotherapy, which is itself associated with cognitive impairment risk [16, 34, 35]. Unfortunately, it is impossible to comment on the differential contributions of treatment regimens to cognitive functioning at this sample size. Nevertheless, these data present a "real-world" view of treatment pathways that will be better reflective of outcomes encountered in series outside of clinical research studies. Finally, there are inevitable issues surrounding practice effects when using the same cognitive assessment tools over time in the same population $[2,4]$. A balance is required between the direct comparability of assessments at different time points and the potential for practice effects.

\section{Conclusions}

Cognitive function in patients with diffuse glioma during early treatment is a complex and dynamic interplay between multiple factors. Both traditional and app-based assessments have complementary roles in understanding cognitive function. This work provides the framework for robust, objective, and accessible assessment across multiple centers. Such data will be useful not only clinically at the individual level, but also for generating the necessary large datasets required to better understand cognitive outcomes in general.

Supplementary Information The online version contains supplementary material available at https://doi.org/10.1007/s00701-022-05162-5.

Acknowledgements We thank all patients for their generous involvement in the study. We also thank Luca Villa, Rohit Sinha, and Jessica Ingham for their contribution to the study. RRG is funded by a Guarantors of Brain Post-Doctoral Fellowship award, by a Cancer Research UK Cambridge Centre RG86786 (CRUK grant ref: A25117) grant, and by the EMERGIA Junta de Andalucía program. Y. E. is funded by a Royal Society Dorothy Hodgkin Research Fellowship. M. A. is funded by the Cambridge Trust-Yousef Jameel Scholarship. S. J. P. is supported by the National Institute for Health Career Development Fellowship (CDF-2018-11-ST2-003). This report is an independent research supported by the National Institute of Research (NIHR Career Development Fellowship, Mr. Stephen Price, CDF-2018-11-ST2-003). M. G. H. received an award from The Brain Tumour Charity (ref: RG86218) to fund this work.

Availability of data and material In accordance with ethics requirements, data will be made available to collaborating centers upon reasonable request.

Code availability Code will be made available upon request. 


\section{Declarations}

Conflict of interest The authors declare no competing interests.

Disclaimer The views expressed in this publication are those of the authors and not necessarily those of the NHS, the National Institute for Health Research or the Department of Health and Social Care.

Open Access This article is licensed under a Creative Commons Attribution 4.0 International License, which permits use, sharing, adaptation, distribution and reproduction in any medium or format, as long as you give appropriate credit to the original author(s) and the source, provide a link to the Creative Commons licence, and indicate if changes were made. The images or other third party material in this article are included in the article's Creative Commons licence, unless indicated otherwise in a credit line to the material. If material is not included in the article's Creative Commons licence and your intended use is not permitted by statutory regulation or exceeds the permitted use, you will need to obtain permission directly from the copyright holder. To view a copy of this licence, visit http://creativecommons.org/licenses/by/4.0/.

\section{References}

1. Anderson SI, Taylor R, Whittle IR (1999) Mood disorders in patients after treatment for primary intracranial tumours. Br J Neurosurg. https://doi.org/10.1080/02688699943295

2. Barron AB, Hebets EA, Cleland TA, Fitzpatrick CL, Hauber ME, Stevens JR (2015) Embracing multiple definitions of learning. Trends Neurosci. https://doi.org/10.1016/j.tins.2015.04.008

3. Benassi M, Garofalo S, Ambrosini F, Sant'Angelo RP, Raggini R, De Paoli G, Ravani C, Giovagnoli S, Orsoni M, Piraccini G (2020) Using two-step cluster analysis and latent class cluster analysis to classify the cognitive heterogeneity of cross-diagnostic psychiatric inpatients. Front Psychol. https://doi.org/10.3389/fpsyg.2020. 01085

4. Cacciamani F, Salvadori N, Eusebi P, Lisetti V, Luchetti E, Calabresi P, Parnetti L (2018) Evidence of practice effect in CANTAB spatial working memory test in a cohort of patients with mild cognitive impairment. Appl Neuropsychol: Adult. https://doi.org/ 10.1080/23279095.2017.1286346

5. Crawford JR, Garthwaite PH (2007) Using regression equations built from summary data in the neuropsychological assessment of the individual case. Neuropsychology. https://doi.org/10.1037/ 0894-4105.21.5.611

6. Demeyere N, Riddoch MJ, Slavkova ED, Bickerton WL, Humphreys GW (2015) The Oxford Cognitive Screen (OCS): validation of a stroke-specific short cognitive screening tool. Psychol Assess. https://doi.org/10.1037/pas0000082

7. Doyle AE, Biederman J, Seidman LJ, Weber W, Faraone SV (2000) Diagnostic efficiency of neuropsychological test scores for discriminating boys with and without attention deficit-hyperactivity disorder. J Consult Clin Psychol. https://doi.org/10.1037/ 0022-006X.68.3.477

8. Duffau H (2005) Lessons from brain mapping in surgery for lowgrade glioma: insights into associations between tumour and brain plasticity. Lancet Neurol. https://doi.org/10.1016/S1474-4422(05) 70140-X

9. Ek L, KristoffersenWiberg M, Vestberg S (2018) Decline in executive functions and speed in suspected low-grade gliomas: a 3-year follow-up of a clinical cohort. Appl Neuropsychol: Adult. https://doi.org/10.1080/23279095.2017.1316506

10. Erez Y, Assem M, Coelho P, Romero-Garcia R, Owen M, McDonald A, Woodberry E, Morris RC, Price SJ, Suckling J, Duncan J,
Hart MG, Santarius T (2020) Intraoperative mapping of executive function using electrocorticography for patients with low-grade gliomas. Acta Neurochir 163(5):1299-1309. https://doi.org/10. 1007/s00701-020-04646-6

11. Harris RJ, Bookheimer SY, Cloughesy TF, Kim HJ, Pope WB, Lai A, Nghiemphu PL, Liau LM, Ellingson BM (2014) Altered functional connectivity of the default mode network in diffuse gliomas measured with pseudo-resting state fMRI. J Neurooncol 116(2):373-379. https://doi.org/10.1007/s11060-013-1304-2

12. Hart MG, Romero-Garcia R, Price SJ, Suckling J (2019) Global effects of focal brain tumors on functional complexity and network robustness: a prospective cohort study. Clin Neurosurg. https://doi. org/10.1093/neuros/nyy378

13. Kaleita TA, Wellisch DK, Cloughesy TF, Ford JM, Freeman D, Belin TR, Goldman J (2004) Prediction of neurocognitive outcome in adult brain tumor patients. J Neurooncol. https://doi.org/ 10.1023/B:NEON.0000021900.29176.58

14. Kroenke K, Spitzer RL, Williams JBW (2001) The PHQ-9: Validity of a brief depression severity measure. J Gen Intern Med. https://doi.org/10.1046/j.1525-1497.2001.016009606.x

15. Lang S, Gaxiola-Valdez I, Opoku-Darko M, Partlo LA, Goodyear BG, Kelly JJP, Federico P (2017) Functional connectivity in frontoparietal network: indicator of preoperative cognitive function and cognitive outcome following surgery in patients with glioma. World Neurosurg. https://doi.org/10.1016/j.wneu.2017.05.149

16. Lawrie TA, Gillespie D, Dowswell T, Evans J, Erridge S, Vale L, Kernohan A, Grant R (2019) Long-term neurocognitive and other side effects of radiotherapy, with or without chemotherapy, for glioma. Cochrane Database Syst Rev. https://doi.org/10.1002/ 14651858.cd013047.pub2

17. Lovejoy DW, Ball JD, Keats M, Stutts ML, Spain EH, Janda L, Janusz J (1999) Neuropsychological performance of adults with attention deficit hyperactivity disorder (ADHD): diagnostic classification estimates for measures of frontal lobe/executive functioning. J Int Neuropsychol Soc. https://doi.org/10.1017/S1355 617799533055

18. Mancuso M, Demeyere N, Abbruzzese L, Damora A, Varalta V, Pirrotta F, Antonucci G, Matano A, Caputo M, Caruso MG, Pontiggia GT, Coccia M, Ciancarelli I, Zoccolotti P, Beni C, Giovannelli F, Ciancarelli I, Zampolini M, Benedetti A, ... Soda M (2018) Using the Oxford cognitive screen to detect cognitive impairment in stroke patients: a comparison with the Mini-Mental State Examination. Front Neurol. https://doi.org/10.3389/fneur. 2018.00101

19. Meyers CA, Hess KR (2003) Multifaceted end points in brain tumor clinical trials: cognitive deterioration precedes MRI progression. Neuro-Oncology. https://doi.org/10.1215/15228 517-5-2-89

20. Meyers JE, Zellinger MM, Kockler T, Wagner M, Miller RM (2013) A validated seven-subtest short form for the WAIS-IV. Appl Neuropsychol: Adult. https://doi.org/10.1080/09084282. 2012.710180

21. Morris R, Blashfield R, Satz P (1981) Neuropsychology and cluster analysis: potentials and problems. J Clin Neuropsychol. https:// doi.org/10.1080/01688638108403115

22. Ng JCH, See AAQ, Ang TY, Tan LYR, Ang BT, King NKK (2019) Effects of surgery on neurocognitive function in patients with glioma: a meta-analysis of immediate post-operative and long-term follow-up neurocognitive outcomes. J Neurooncol. https://doi.org/10.1007/s11060-018-03023-9

23. Perugini EM, Harvey EA, Lovejoy DW, Sandstrom K, Webb AH (2000) The predictive power of combined neuropsychological measures for attention-deficit/hyperactivity disorder in children. Child Neuropsychol. https://doi.org/10.1076/chin.6.2.101.7059

24. Quental NBM, Brucki SMD, Bueno OFA (2013) Visuospatial function in early Alzheimer's disease-the use of the visual object 
and space perception (VOSP) battery. PLoS ONE. https://doi.org/ 10.1371/journal.pone.0068398

25. Reijneveld JC, Taphoorn MJB, Coens C, Bromberg JEC, Mason WP, Hoang-Xuan K, Ryan G, Hassel MB, Enting RH, Brandes AA, Wick A, Chinot O, Reni M, Kantor G, Thiessen B, Klein M, Verger E, Borchers C, Hau P, ... Baumert BG (2016) Healthrelated quality of life in patients with high-risk low-grade glioma (EORTC 22033-26033): a randomised, open-label, phase 3 intergroup study. Lancet Oncol. https://doi.org/10.1016/S14702045(16)30305-9

26. Rofes A, Mandonnet E, Godden J, Baron MH, Colle H, Darlix A, de Aguiar V, Duffau H, Herbet G, Klein M, Lubrano V, Martino J, Mathew R, Miceli G, Moritz-Gasser S, Pallud J, Papagno C, Rech F, Robert E, ... Wager M (2017) Survey on current cognitive practices within the European Low-Grade Glioma Network: towards a European assessment protocol. Acta Neurochir. https:// doi.org/10.1007/s00701-017-3192-2

27. Romero-Garcia R, Erez Y, Oliver G, Owen M, Merali S, Poologaindran A, Morris RC, Price SJ, Santarius T, Suckling J, Hart MG (2020) Practical application of networks in neurosurgery: combined 3-dimensional printing, neuronavigation, and preoperative surgical planning. World Neurosurg 137:e126-e137. https:// doi.org/10.1016/j.wneu.2020.01.085

28. Satoer D, Visch-Brink E, Dirven C, Vincent A (2016) Glioma surgery in eloquent areas: can we preserve cognition? Acta Neurochirurgica. https://doi.org/10.1007/s00701-015-2601-7

29. Spitzer RL, Kroenke K, Williams JBW (1999) Validation and utility of a self-report version of PRIME-MD: The PHQ Primary Care Study. J Am Med Assoc. https://doi.org/10.1001/jama.282. 18.1737

30. Taphoorn MJB, Klein M (2004) Cognitive deficits in adult patients with brain tumours. Lancet Neurol. https://doi.org/10. 1016/S1474-4422(04)00680-5

31. van Dellen E, Douw L, Hillebrand A, Ris-Hilgersom I, Schoonheim M, Baayen J, de Witt Hamer P, Velis D, Klein M, Heimans
J, Stam C (2012) MEG network differences between low- and high-grade glioma related to epilepsy and cognition. PLoS ONE 7(11):e50122

32. van der Hurk PR, Hodges JR (1995) Episodic and semantic memory in Alzheimer's disease and progressive supranuclear palsy: a comparative study. J Clin Exp Neuropsychol. https://doi.org/10. 1080/01688639508405137

33. Vlaar AMM, Wade DT (2003) The adult memory and information processing battery (AMIPB) test of information-processing speed: a study of its reliability and feasibility in patients with multiple sclerosis. Clin Rehabil. https://doi.org/10.1191/0269215503cr625 oa

34. Wefel JS, Cloughesy T, Zazzali JL, Zheng M, Prados M, Wen PY, Mikkelsen T, Schiff D, Abrey LE, Alfred Yung WK, Paleologos N, Nicholas MK, Jensen R, Vredenburgh J, Das A, Friedman HS (2011) Neurocognitive function in patients with recurrent glioblastoma treated with bevacizumab. Neuro Oncol. https://doi.org/ 10.1093/neuonc/nor024

35. Wefel JS, Noll KR, Rao G, Cahill DP (2016) Neurocognitive function varies by IDH1 genetic mutation status in patients with malignant glioma prior to surgical resection. Neuro Oncol 18(12):1656-1663. https://doi.org/10.1093/neuonc/now165

36. Wilson B, Emslie H, Foley J, Shiel A, Watson P, Hawkins K, Al E (2005) Cambridge Prospective Memory Test (CAMPROMPT). Harcourt Assessment. Harcourt Assessment, London

37. Zhou X, Grégoire J, Zhu J (2010) WAIS-IV clinical use and interpretation. In: WAIS-IV Clinical Use and Interpretation.https://doi. org/10.1016/B978-0-12-375035-8.10005-9

Publisher's note Springer Nature remains neutral with regard to jurisdictional claims in published maps and institutional affiliations.

\title{
Authors and Affiliations
}

\author{
Rafael Romero-Garcia ${ }^{1,2} \mathbb{1} \cdot$ Mallory Owen ${ }^{1} \cdot$ Alexa McDonald $^{3} \cdot$ Emma Woodberry $^{3} \cdot$ Moataz Assem $^{4}$. \\ Pedro Coelho ${ }^{5} \cdot$ Rob C. Morris $^{6} \cdot$ Stephen J. Price ${ }^{6} \cdot$ Tom Santarius $^{6,7}$. John Suckling ${ }^{1,8,9} \cdot$ Tom Manly $^{9} \cdot$ Yaara Erez $^{4,10}$. \\ Michael G. Hart ${ }^{1}$
}

1 Department of Psychiatry, University of Cambridge, Herchel Smith Building for Brain and Mind Sciences, Robinson Way, Cambridge CB2 0SZ, UK

2 Dpto. de Fisiología Médica Y Biofísica, Instituto de Biomedicina de Sevilla (IBiS) HUVR/CSIC, Universidad de Sevilla, Seville, Spain

3 Department of Neuropsychology, Cambridge University Hospitals NHS Foundation Trust, Cambridge, UK

4 MRC Cognition and Brain Sciences Unit, University of Cambridge, Cambridge, UK

5 Neurophys Limited, Cambridge, UK
6 Department of Neurosurgery, Addenbrooke's Hospital, Cambridge, UK

7 Physiology, Development and Neuroscience, University of Cambridge, Cambridge, UK

8 Behavioural and Clinical Neuroscience Institute, University of Cambridge, Cambridge, UK

9 Cambridge and Peterborough NHS Foundation Trust, Cambridgeshire, UK

10 Faculty of Engineering, Bar-Ilan University, Ramat-Gan, Israel 